\title{
The Intramolecular Pressure and the Extension of the Critical Point's Influence Zone on the Order Parameter
}

\author{
Jose Luis Rivera, Homero Nicanor-Guzman, and Roberto Guerra-Gonzalez \\ Facultad de Ingeniería Química, Universidad Michoacana de San Nicolás de Hidalgo, 58000 Morelia, MICH, Mexico
}

Correspondence should be addressed to Jose Luis Rivera; rivera_jose_l@yahoo.com

Received 20 April 2015; Revised 23 July 2015; Accepted 26 July 2015

Academic Editor: Sergei Sergeenkov

Copyright (C) 2015 Jose Luis Rivera et al. This is an open access article distributed under the Creative Commons Attribution License, which permits unrestricted use, distribution, and reproduction in any medium, provided the original work is properly cited.

The critical point affects the coexistence behavior of the vapor-liquid equilibrium densities. The length of the critical influence zone is under debate because for some properties, like shear viscosity, the extension is only a few degrees, while for others, such as the density order parameter, the critical influence zone covers up to hundreds of degrees below the critical temperature. Here we show that, for ethane, the experimental critical influence zone covers a wide zone of tens of degrees (below the critical temperature) down to a transition temperature, at which the apparent critical influence zone vanishes, and the transition temperature can be predicted through a pressure analysis of the coexisting bulk liquid phase, using a simple molecular potential. The liquid phases within the apparent critical influence zone show low densities, making them behave internally like their corresponding vapor phases. Therefore, Molecular Dynamics simulations reveal that the experimentally observed wide extension of the critical influence zone is the result of a vapor-like effect due to low bulk liquid phase densities.

\section{Introduction}

Vapor-liquid equilibrium (VLE) for pure components is present between the triple and critical points; from a transition temperature between these two points (closer to the critical point) up to the critical point (critical influence zone), equilibrium liquid phases start to behave more like their corresponding vapor phases [1], characterized by larger density changes than those close to the triple point for the same temperature changes. In the critical influence zone (CIZ), the coexisting vapor and liquid densities approach asymptotically, indicating a strong influence of the critical behavior on the coexisting densities, and the universal power law describes their difference:

$$
\Delta \rho=B_{0} \tau^{\beta},
$$

where $\Delta \rho=\rho_{l}-\rho_{v}$ is the order parameter of phase transition, $\rho_{l}$ and $\rho_{v}$ represent the liquid and vapor coexisting densities, respectively, $\tau=\left(T_{C}-T\right) / T_{C}, T_{C}$ is the critical temperature, $\beta$ is a universal critical exponent, and $B_{0}$ is a constant dependent on the fluid. Below the transition temperature (down to the triple point), $\Delta \rho$ deviates from the universal power law, and the correct value of $\Delta \rho$ can be calculated by the introduction of nonasymptotic corrections $[2,3]$. Water order parameters (experimental) in the CIZ show a linear behavior and follow (1) down to $\sim 130^{\circ}$ below $T_{C}$ (transition temperature). In comparison, a pure Lennard-Jones fluid in the CIZ follows the universal power law for almost all its coexistence region [4]. The wide influence zone in the order parameter is unexpected, since the critical influence on other properties (shear viscosity) only covers a few degrees below $T_{C}[4,5]$, and a different phenomenon (other than the critical point influence) is affecting the length of the apparent CIZ. Clearly, the main difference between the Lennard-Jones fluid and water is the nature and length of the intermolecular interactions present in the water molecule, but, in this study, we study a second difference between these two systems, which is the presence of intramolecular interactions in the water molecule, not present in the Lennard-Jones fluid, but the study based on intramolecular forces is not completely independent of a study based on intermolecular forces.

Intra- and intermolecular (pair effective) forces are not completely independent in equilibrium liquid phases; for a specific fluid, its liquid phases in equilibrium close to the triple point will have in average more compressed molecules (higher compressive intramolecular forces) than 
liquid phases in equilibrium near the critical point, which will have lower densities, and therefore the molecules are less compressed (lower compressive or even expansive intramolecular forces). On the other hand, molecules in liquid phases close to the triple point will show stronger intermolecular forces, as those systems are denser than systems at higher temperatures. Therefore, based only on the monotonic decrement of the equilibrium liquid densities from the triple to the critical point, both intra- and intermolecular forces should also monotonically decrease in this coexistence region.

In this paper we study the intramolecular forces in the pressure profiles of vapor-liquid systems of ethane under equilibrium for the whole range of equilibrium coexistence and correlate the changes in the bulk liquid pressure profiles to their corresponding order parameters in the critical influence zone to delimitate its range of influence. The remainder of the paper is organized as follows: in Section 2, we provide details of the molecular models, and the molecular simulation methodologies used to produce the results discussed in Section 3, and finally we report the conclusions in Section 4.

\section{Methodology}

To understand the unexpected range of the critical-influence in the order parameter, we analyzed through Molecular Dynamics (MD) simulations the ethane molecule, which compared to a Lennard-Jones fluid shows intramolecular interactions as the water molecule, but it has a less complex intermolecular potential than the water molecule. For the ethane molecule, experimental values of $\Delta \rho$ as a function of $\tau$ have been plotted in Figure 1. The critical behavior apparently influences $\Delta \rho$ for $\sim 75^{\circ}$, between a transition temperature $\sim 230 \mathrm{~K}(\tau \sim-1.4$, Figure 1$)$ and the $T_{C}$. For this range of temperatures, $\Delta \rho$ values show good agreement with the universal power law (1).

MD simulations allow the study of systems under phase equilibrium and specifically can be applied to examine the contributions due to intramolecular and intermolecular interactions to the thermophysical properties directly at the equilibrium interface and also at their corresponding bulk phases [10-14]. The simulation of the coexisting VLE uses pair-effective intermolecular potentials that are capable of reproducing their thermophysical properties $[7,15,16]$ and transport properties for simple fluids [17-19]. The VLE of ethane was simulated using the MD methodology (in-house code) in the range of $100-260 \mathrm{~K}$. The simulations were carried out using the Verlet algorithm with a Nose thermostat [20] and a time step of $1 \mathrm{fs}$. A cutoff radius of 4.5 sigmas $(17.0235 \AA)$ was employed, which is long enough to avoid the use of long-range corrections [21]. Some points were recalculated using longer cutoff radius (5.5 sigmas) to verify the employed cutoff radius. The initial system consists of a cubic cell of ethane molecules surrounded by three empty rectangular cells, which produces a simulation cell 4 times longer in the inhomogeneous direction (Figure 2, top). The dimensions of the combined simulation cell are $43.7 \times 43.7 \times 174.8 \AA^{3}$, and the cell contains 1000 molecules of ethane. We use $10 \mathrm{~ns}$

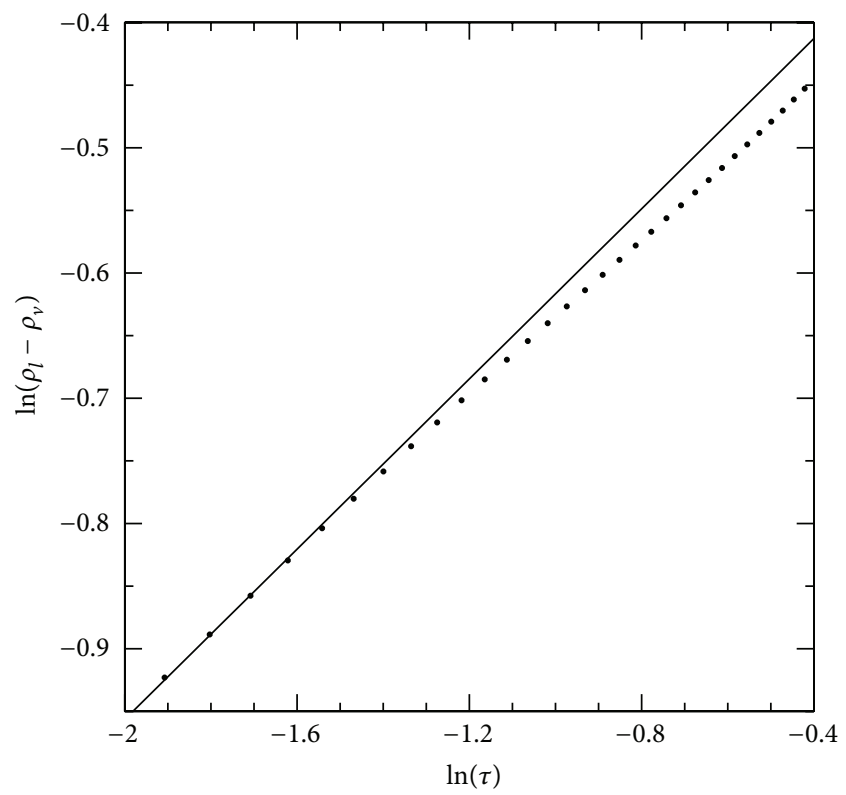

FIgURE 1: Plot of the order parameter $\Delta \rho=\rho_{l}-\rho_{v}$ versus the reduced temperature $\tau$ for experimental ethane [6] (solid circles), where $\rho_{l}$ and $\rho_{v}$ represent the liquid and vapor coexisting densities. The straight solid line represents the fitted results for $\Delta \rho$ using the scaling equation (1).

to generate statistical averages of the properties, following an initial run of $1 \mathrm{~ns}$ for equilibration. To equilibrate the system, it was slowly heated, starting from a configuration at $0 \mathrm{~K}$, to eliminate translation of the "liquid slab" of ethane through the simulation cell. To avoid oscillations problems in the calculated properties, equilibration was carried out using the Canonical ensemble, while production was carried out at the microcanonical ensemble [22]. Density and pressure profiles were calculated using the standard expressions reported elsewhere $[14,23]$. The interaction potential for ethane molecules was the flexible version of the MIE potential [7], which in its rigid version reproduces well the coexistence properties, including the coexisting densities, critical point, and vaporization enthalpy [7]. The inclusion of flexible bonds in the MIE potential does not affect their ability to reproduce their coexistence properties [7]. The vibrational bond expression used in this work is a quadratic harmonic oscillator, similar to the one used in the flexible TraPPE potential [16].

\section{Results}

MD simulations in VLE for ethane show high- and lowdensity coexisting phases divided by interfaces (Figure 2, top). Density profiles can be obtained from time averages on mechanically stable configurations to define the bulk and interfacial zones (Figure 2, bottom). The density profiles show 3 distinctive zones: the bulk vapor and liquid phases and their corresponding interfaces. At $100 \mathrm{~K}$, close to the triple point, the density profile shows marked oscillations in the bulk liquid phase, resembling the behavior of a solid, which have 


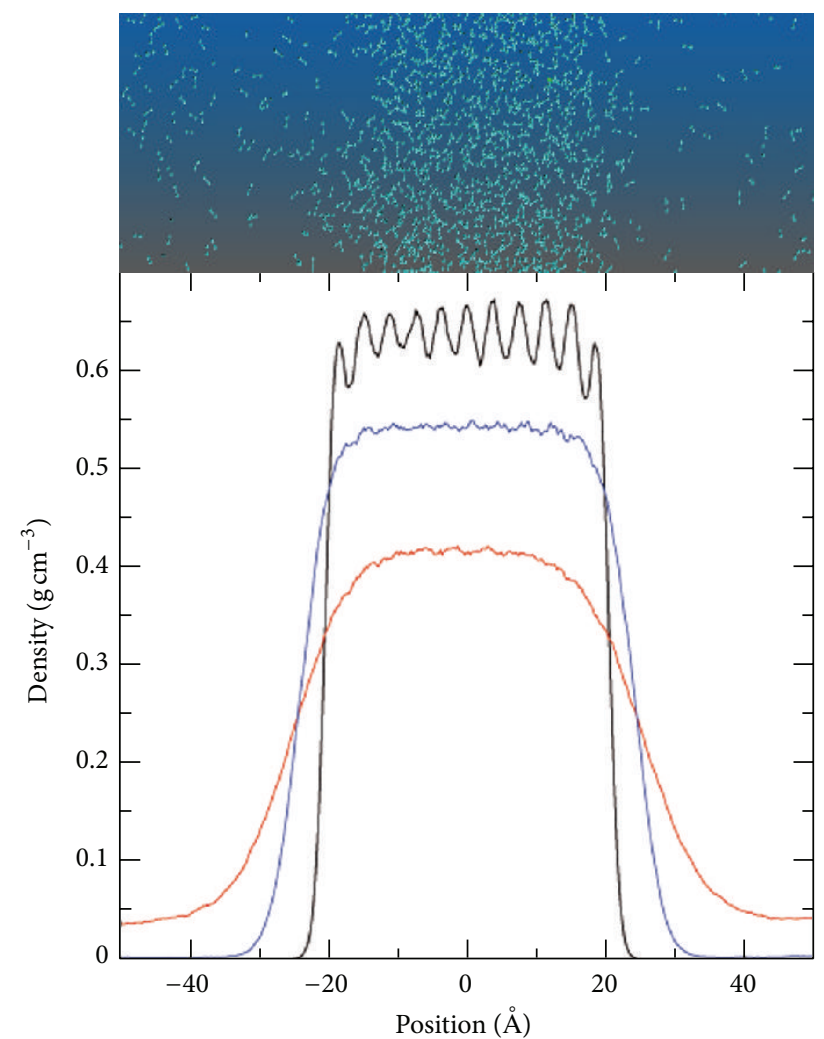

FIGURE 2: The top figure represents an instantaneous snapshot of the ethane molecules (flexible MIE potential) in the vapor-liquid equilibrium at $260 \mathrm{~K}$. The $x$-axis represents the inhomogeneous direction, while the $y$-axis represents one of the two homogeneous directions. The bottom figure shows density profiles of ethane in the VLE as a function of the simulation cell position in the inhomogeneous direction of the simulation cell. The black line represents the profile at $100 \mathrm{~K}$, while blue and red represent the profiles at $180 \mathrm{~K}$ and $260 \mathrm{~K}$, respectively.

been observed previously with other potentials [8] and also described experimentally [1]. The bulk coexisting densities from the simulations results using the flexible MIE potential used in this work [7] reproduce well the experimental coexistence behavior [6] (Figure 3), with a small underprediction of the critical temperature $\left(\sim 4.5^{\circ}\right)$, while the rigid version of the MIE potential produces a small overprediction of the critical temperature $\left(\sim 3^{\circ}\right)$. Lennard-Jones based models reproduce as well the coexisting curve $[8,9]$.

The influence of the intramolecular interactions on the length of the critical-like behavior can be studied through an analysis of the bulk pressures in the VLE for a simple molecular potential of ethane. The virial expression of the pressure tensor can be divided into three contributions:

$$
\begin{aligned}
P_{\alpha}= & (\text { kinetic })_{\alpha}+(\text { intermolecular potential })_{\alpha} \\
& +(\text { intramolecular potential })_{\alpha},
\end{aligned}
$$

where $P_{\alpha}$ represents the normal $\left(P_{N}\right)$ or tangential pressure $\left(P_{T}\right)$. Complete expressions of the pressure tensors in each direction have been published previously and can be found

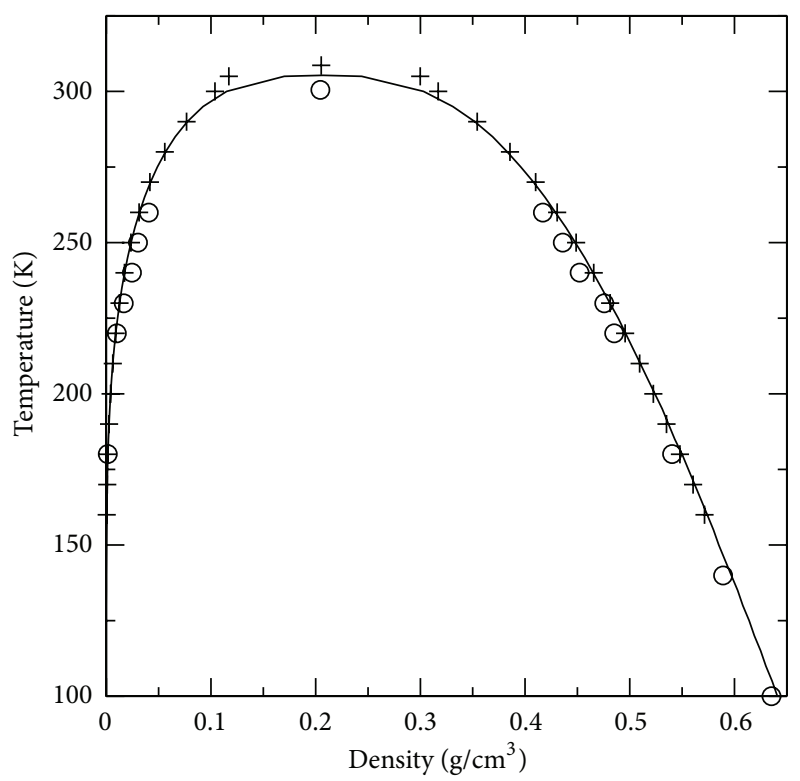

FIgURE 3: Coexistence densities curve of ethane in the vapor-liquid equilibrium. The continuous line represents the experimental results [6]. Plus symbols represent Monte Carlo simulations results for the rigid MIE potential [7]. Circles represent Molecular Dynamics simulations results using the flexible MIE potential used in this work.

elsewhere [14]. Figure 4 shows simulation results of the position profiles of the pressure contributions and total pressure profiles in the inhomogeneous direction $\left(P_{N}\right)$ and the homogeneous direction $\left(P_{T}\right)$ at $100 \mathrm{~K}$. As expected, kinetic contributions in both directions show the same profile, similar to the density profiles; the main contributions are due to the momentum of molecules in the bulk liquid phase and their adjacent interfaces. Intramolecular profiles show similar profiles to the kinetic profiles, but the intramolecular profiles show only contributions in the bulk liquid phase. The fact that the intramolecular profiles in both directions are shorter than those of the kinetic contributions shows that the intramolecular contributions are mainly the result of vibrating molecules at the bulk liquid phase, but not at the interfaces, revealing that molecules at the interfaces are moving free of interactions that compress or expand its intramolecular bond. The intermolecular contributions show the typical behavior; in the normal direction interacting molecules at the bulk liquid interface are the main contributions to the profile, while in the tangential direction the profiles show peaks at the interfaces, which are the main contribution to the surface tension, while at the bulk liquid phase the contributions are similar to those present in the normal profile. The concept of macroscopic pressure is commonly associated with intermolecular forces, but, at the molecular level, the inclusion of intramolecular contributions to the total pressure profile accomplishes the requirement of mechanical equilibrium, making the total pressure profiles in the bulk vapor and bulk liquid phases equal (Figure 4).

The effect of temperature on the pressure profiles was studied simulating the systems at temperatures between 100 


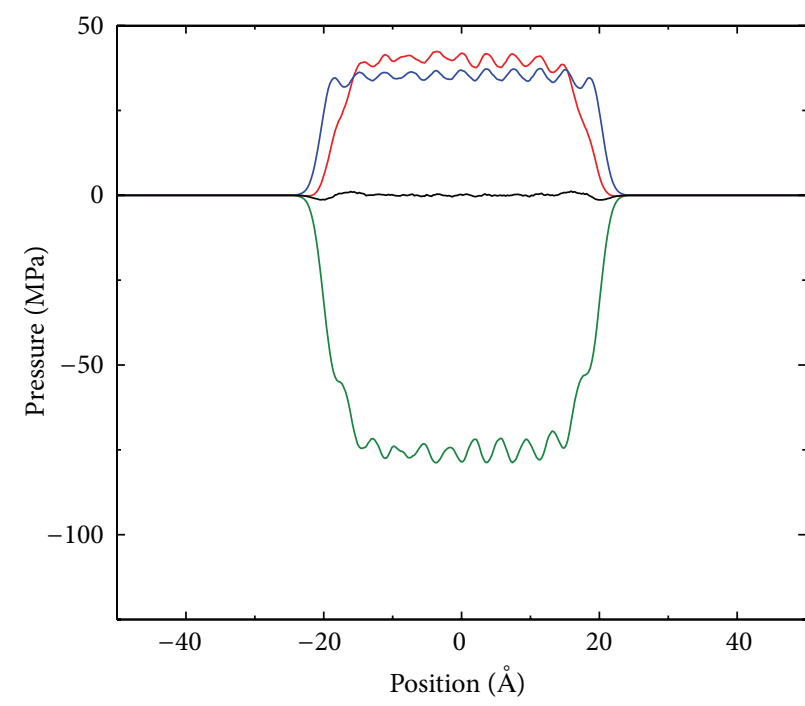

(a)

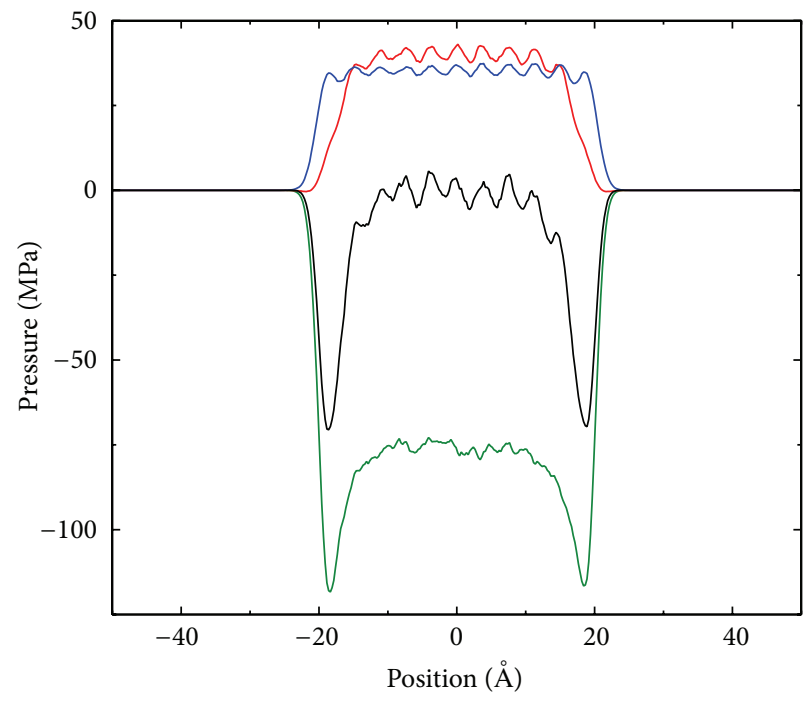

(b)

Figure 4: Profiles of the contributions to the normal (a) and tangential pressures (b), for ethane in the VLE at $100 \mathrm{~K}$ as a function of the simulation cell position in the inhomogeneous direction of the simulation cell. Kinetic, intramolecular, and intermolecular contributions for ethane are represented in the figure by blue, red, and green profiles, respectively. Black profiles represent the total pressure in the respective direction.

and $260 \mathrm{~K}$. Kinetic pressure profiles show the same behavior in the normal and tangential profiles (Figures 5(a) and 5(b)). From 100 to $200 \mathrm{~K}$ the kinetic contribution grows with the temperature in the bulk liquid phase, but from $200 \mathrm{~K}$ to $240 \mathrm{~K}$ the contribution does not grow, which is explained later in this section in terms of the dependence of the profile on other thermophysical properties and profiles. Intermolecular pressure profiles are shown in Figures 5(e) and 5(f) for $P_{N}$ and $P_{T}$, respectively. In the coexisting bulk liquid phase (CBLP), negative intermolecular contributions are the result of net cohesive forces, and the average pressure decreases as the temperature increases. In the coexisting bulk vapor, the intermolecular contributions are insignificant due to long separations of the molecules in the vapor phase. At the interface, the pronounced peaks at low temperatures $(100$ and $140 \mathrm{~K})$ in the tangential contributions are the main contributors to the surface tension. Surface tension results (Figure 6) using the MIE potential show good agreement with experimental results [6] at moderate and high temperatures, while, at the low temperature of $100 \mathrm{~K}$, there is a small deviation, but lower than other simple ethane models like OPLS and TraPPE $[8,9]$.

Figures 5(c) and 5(d) show the intramolecular, normal, and tangential pressure profiles, respectively; at low temperatures, the average intramolecular contribution in the CBLP is positive indicating that the majority of molecular states show compressed bond separations, which can be expected for a normal liquid state, but, as the temperature increases, the average magnitude of the intramolecular contribution decreases, taking negative average values at higher temperatures. Negative intramolecular pressures are also found in the average magnitude of the coexisting bulk vapor phase, and they probably indicate that the majority of molecular states show bond separations with positive deviations from the ground value, which are probably the result of intramolecular pulling forces acting on the bond atoms, which are the result of net attractive intermolecular interactions, typical of bulk vapor phases.

The intramolecular pressure in the CBLP can be used to establish the transition temperature, at which the CBLPs move from average positive to negative intramolecular pressures (similar to those present in a coexisting bulk vapor phase, Figure 5). Figure 7 shows the average intramolecular pressures in the CBLP as a function of the system temperature. The intramolecular pressure regularly reduces as the temperature increases, almost in a linear fashion. The best fit to a quadratic curve shows the locations of the transition temperature $\sim 224 \mathrm{~K}$. This transition temperature is close to the transition temperature where the CIZ vanishes (Figure 1). In Figure 7 we also observe that the average intermolecular pressure decreases monotonically, and, together with the intramolecular pressure, the total potential pressure shows a minimum between 240 and $250 \mathrm{~K}$. Compared to the potential pressure, the kinetic pressure shows a symmetric behavior, with a maximum also in the range of $240-250 \mathrm{~K}$. The maximum kinetic pressure in the CBLP can be understood if we take into account that the kinetic pressure is a function not only of the increasing temperature, but also of the bulk liquid density, which decreases as the temperature increases, and the decrements are more pronounced at temperatures close to the critical point. Therefore, there should be a maximum in the kinetic pressure when increasing contributions due to temperature increments cannot compete with decreasing contributions due to increasingly lower densities. The reported values for the kinetic, intramolecular, and intermolecular pressures for ethane added into the total liquid 


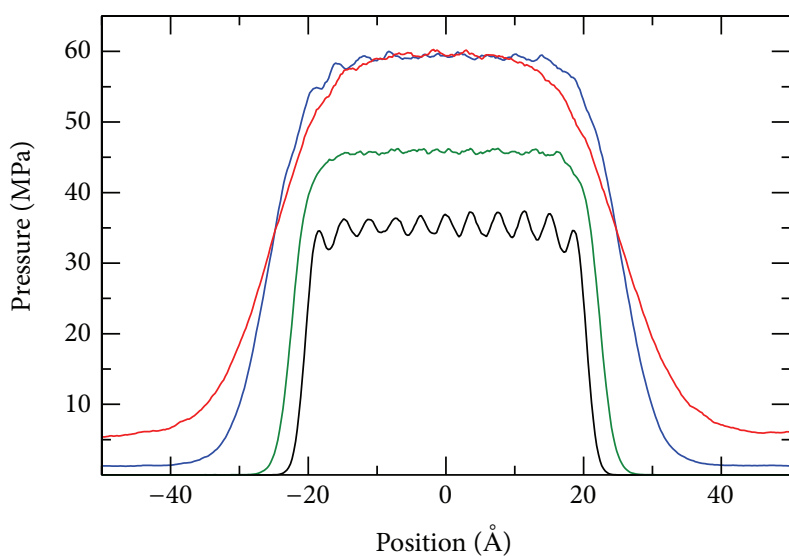

(a)

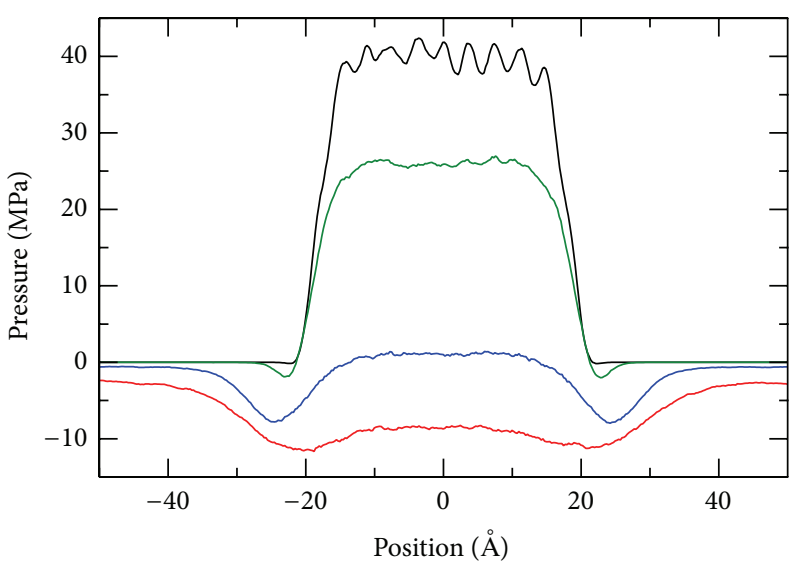

(c)

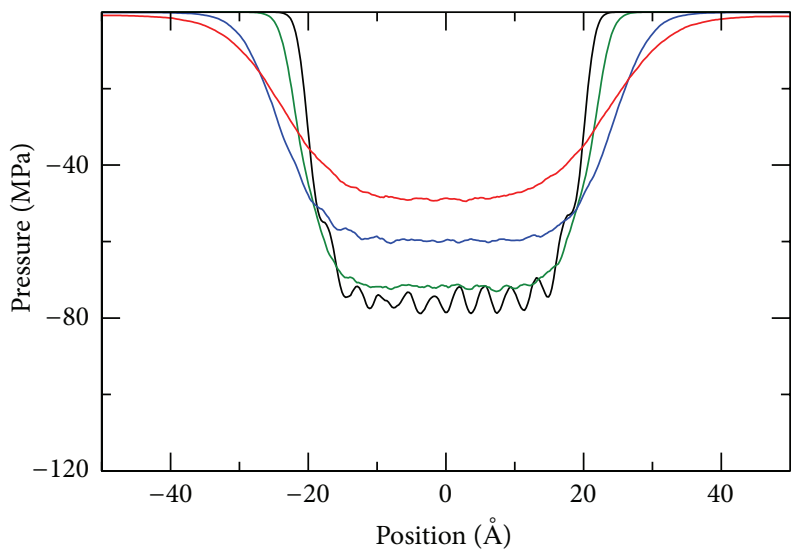

(e)

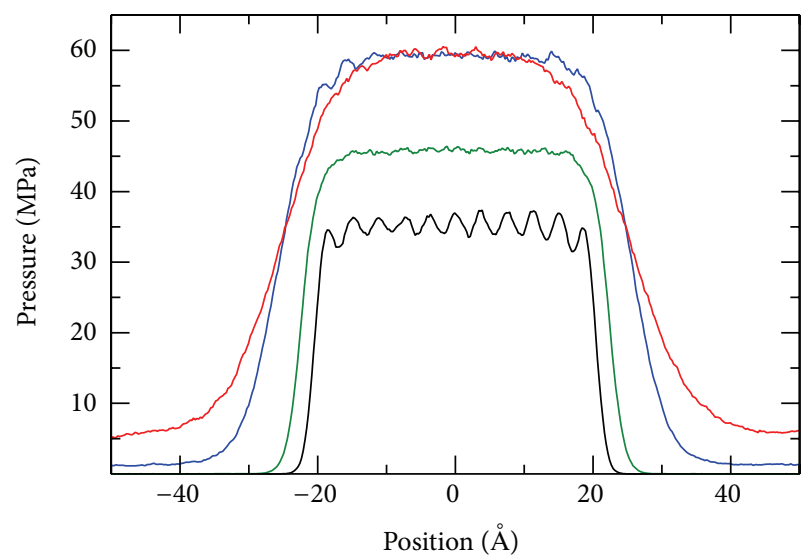

(b)

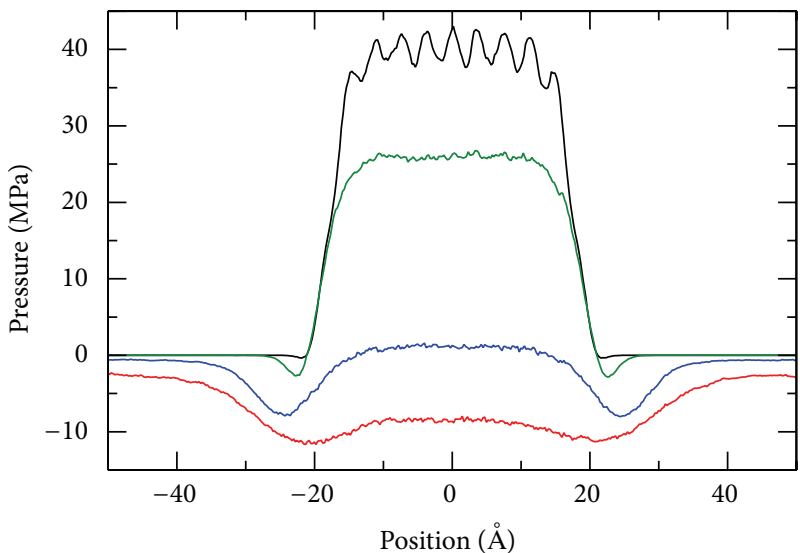

(d)

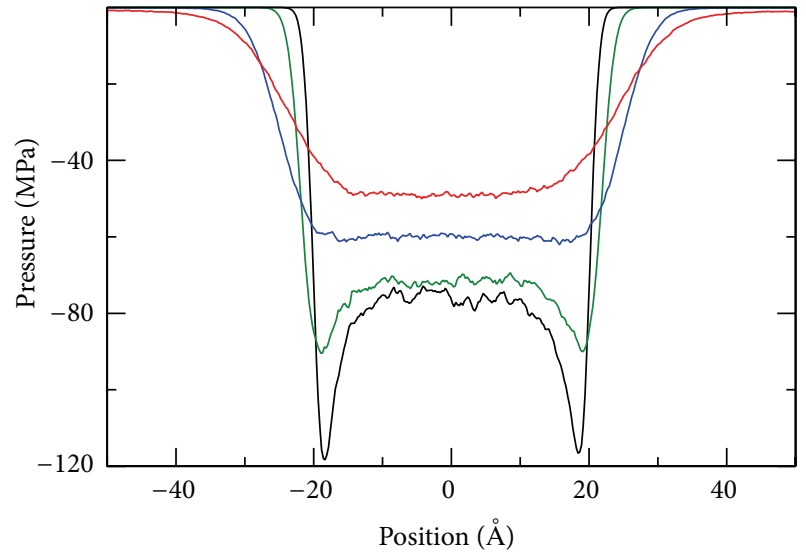

(f)

FIGURE 5: Profiles of the contributions to the normal (left) and tangential pressures (right), for ethane in the VLE as a function of the simulation cell position in the inhomogeneous direction of the simulation cell. The color code in (a)-(f) represents results at $100 \mathrm{~K}$ (black), $140 \mathrm{~K}$ (green), $220 \mathrm{~K}$ (blue), and $260 \mathrm{~K}$ (red). Kinetic contributions are shown in (a) and (b), while intramolecular and intermolecular contributions are shown in (c), (d), and (e) and (f), respectively.

pressure reproduce well the experimental values of the vapor pressure (Figure 9), which for a system under equilibrium should be the same.

The threshold value for the transition temperature where the CIZ vanishes can also be studied more directly using the bond-separation distributions as a function of the temperature. The insight of Figure 8 shows the bond-separation deviation (from the ground value) distributions at a low $(100 \mathrm{~K})$ and a high temperature $(260 \mathrm{~K})$, for the CBLP of ethane in the VLE. As expected, the distributions widen as 


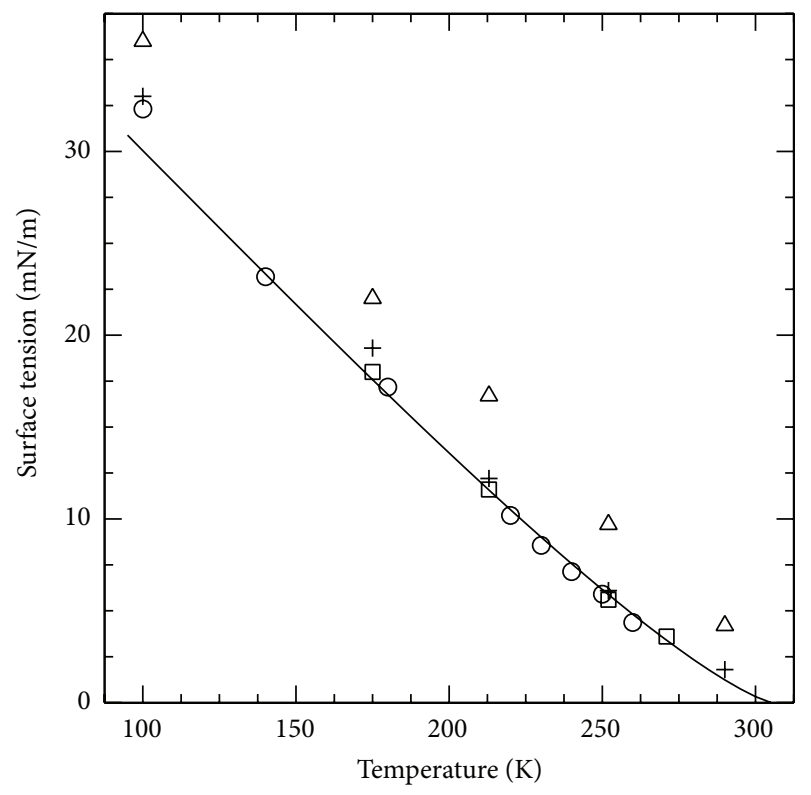

FIGURE 6: Surface tension of ethane as a function of the temperature. The continuous line represents experimental results [6]. Triangle, rectangles, and plus symbols represent simulations results using the OPLS, flexible TraPPE, and rigid TraPPE potentials, respectively $[8,9]$. Circles represent results of this work using the flexible MIE potential.

the temperature increases, but small changes in the median value are apparent. Figure 8 shows the deviation of the median of the bond-separation distributions from the ground value $\left(b_{0}=1.54 \AA\right)$ as a function of the system temperature. The deviations grow from negative values in the range of $100-240 \mathrm{~K}$ to positive values in the range $240-260 \mathrm{~K}$, with a value of zero at $240 \mathrm{~K}$. The transition temperature $(240 \mathrm{~K})$ from negative to positive deviations is also close to the transition temperature where the CIZ vanishes (Figure 1). Negative median deviations are expected in moderate and highly dense phases like compressed liquids and solids, where strong, cohesive forces compress the molecular volume, while positive median deviations are expected in low dense phases (vapors) where atoms in the molecular bond are pulled apart due to net attractive intermolecular forces with other molecules. The net attractive intermolecular forces in low dense phases are the result of long intermolecular separations, typically found in vapors and gasses. A simple calculation also confirms the bond deviations behavior; at the transition temperature $(240 \mathrm{~K})$, the CBLP density produces an average site separation (we have two $\mathrm{CH}_{3}$ sites per ethane molecule) equal to the characteristic separation sigma (MIE potential), where the intermolecular interaction energy is zero; then, for this unique site separation, molecules are packed without any compression or expansion in their bond separation due to intermolecular interactions. To calculate the unique site separation, we consider that, at $240 \mathrm{~K}$, the experimental density of ethane is $0.46508 \mathrm{~g} / \mathrm{cm}^{3}$. For a number of ethane molecules (500), we have $1000 \mathrm{CH}_{3}$ sites or $2.490867 \times$ $10^{-20} \mathrm{~g}$, which correspond to $5.461469 \times 10^{-20} \mathrm{~cm}^{3}$; if we

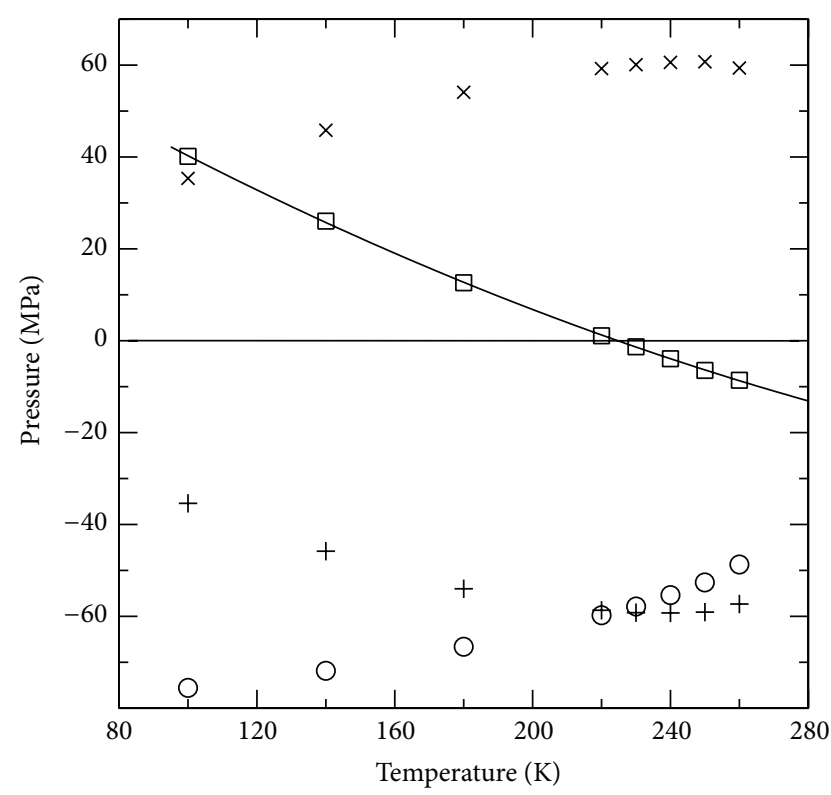

FIGURE 7: Average values of the intramolecular (squares), intermolecular (circles), total potential (sum of the intramolecular and intermolecular pressure, crosses), and kinetic (stars) pressures in the CBLP for ethane, as a function of the system temperature, in the VLE using the flexible MIE potential.

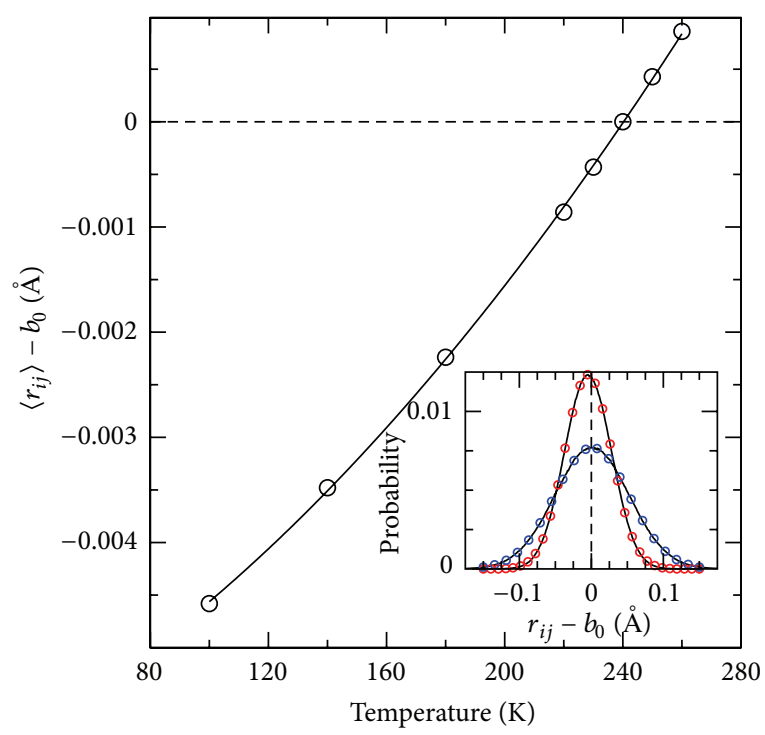

FIgURE 8: Median of the deviations of the bond-separation distributions, for ethane in the VLE (flexible MIE potential) as a function of the system temperature. Bond-separation deviation distributions for ethane in the VLE, at $100 \mathrm{~K}$ (red circles) and $260 \mathrm{~K}$ (blue circles) (insight of Figure 8).

distribute homogeneously the $\mathrm{CH}_{3}$ sites in a simple cubic system, the separation between the $\mathrm{CH}_{3}$ sites is $3.790 \AA$, which is very close to the sigma value of the MIE potential (3.783 ̊).

We also investigated the effect of the cutoff radius, recalculating points in the threshold temperature where the CIZ 


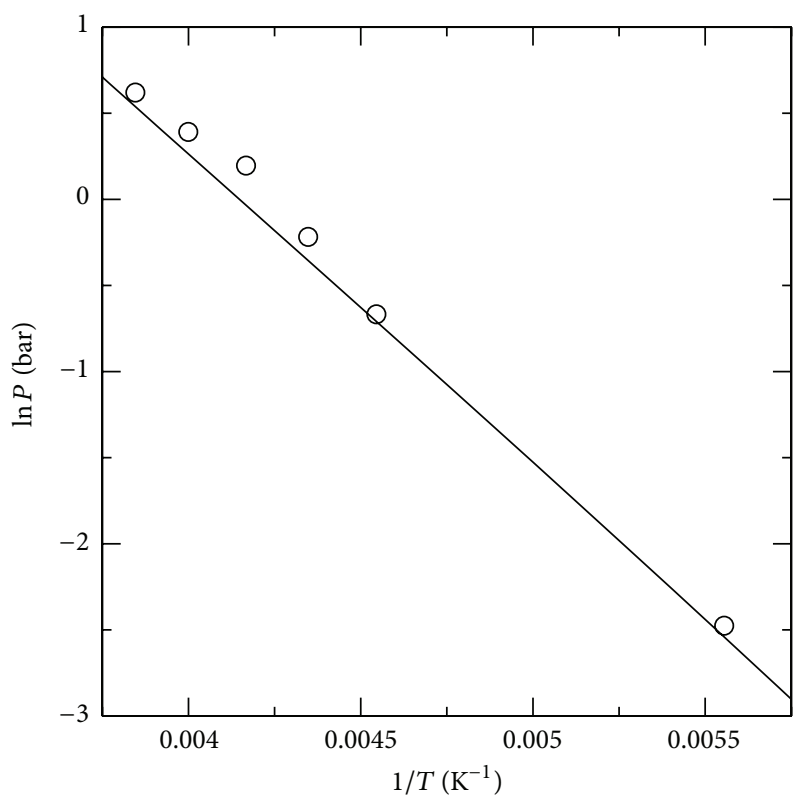

FIGURE 9: Vapor pressures of ethane in the VLE as a function of the system temperature. Continuous line represents experimental results [6]. Circles represent results of this work using the MIE potential [7].

vanishes using longer cutoff radius (5.5 sigmas), and found negligible effects on the determination of the transition temperatures. We were also interested in the effects of the flexibility of the intramolecular potential used and recalculated some points near the threshold temperature where the CIZ vanishes using an intramolecular potential 10 times stiffer, more similar to the behavior of the original MIE potential (rigid), and found that the threshold temperature moved 2 degrees $(\sim 222 \mathrm{~K})$.

\section{Conclusions}

By using Molecular Dynamics simulations, we can predict the transition temperature, which delimits the apparent critical influence zone. We demonstrate that an analysis of the intramolecular pressure in the coexisting bulk liquid phases can be used to predict the extension of the apparent critical influence region. The coexisting bulk liquid phases in the apparent critical influence zone behave more like vapors in terms of its average intramolecular forces. Within the temperatures studied, we found that coexisting bulk liquid phases below the critical influence zone show negative intermolecular pressures and positive intramolecular pressures, while for coexisting bulk liquid phases within the critical influence zone both pressures are negative. For vapor phases, the intramolecular part is negative, and the intermolecular part is also negative and almost zero. Bond distributions confirm the "vapor-like" behavior of coexisting bulk liquid phases in the critical influence zone, which for the ethane molecules extend in the range of $\sim 230 \mathrm{~K}$ to the critical point. Liquids behaving like vapors have also been observed experimentally in calorimetric studies of drops with low densities [24]. Therefore, the apparent wide zone of the experimentally observed critical influence zone is due to two contributions; from the transition temperature to the critical point we have the vapor-like effect due to low coexisting bulk liquid phase densities, while at temperatures in the vicinity of the critical temperature, the critical point effects dominate.

\section{Conflict of Interests}

The authors declare that there is no conflict of interests regarding the publication of this paper.

\section{Acknowledgments}

Jose Luis Rivera thanks CONACYT-Ciencia Básica (México), under Grant no. 134508, and Universidad Michoacana de San Nicolás de Hidalgo for sabbatical support. Homero Nicanor-Guzman thanks UMSNH for a scholarship.

\section{References}

[1] E. Gregersen, "Behaviour of substances near critical and triple points," in The Britannica Guide to Matter, p. 64, Britannica Educational Publishing, New York, NY, USA, 2011.

[2] Y. Guissani and B. Guillot, "A computer simulation study of the liquid-vapor coexistence curve of water," Journal of Chemical Physics, vol. 98, no. 10, pp. 8221-8235, 1993.

[3] F. J. Wegner, "Corrections to scaling laws," Physical Review B, vol. 5, no. 11, pp. 4529-4536, 1972.

[4] I. Brovchenko and A. Oleinikova, "Phase diagram of bulk water," in Interfacial and Confined Water, p. 305, Elsevier, Amsterdam, The Netherlands, 2008.

[5] R. F. Berg, M. R. Moldover, and G. A. Zimmerli, "Viscoelasticity of xenon near the critical point," Physical Review Letters, vol. 82, no. 5, pp. 920-923, 1999.

[6] P. J. Linstrom and W. G. Mallard, "NIST chemistry Webbook," NIST Standard Reference Database 69, National Institute of Standards and Technology, Gaithersburg, Md, USA, 2014, http://webbook.nist.gov.

[7] J. J. Potoff and D. A. Bernard-Brunel, "Mie potentials for phase equilibria calculations: application to alkanes and perfluoroalkanes," The Journal of Physical Chemistry B, vol. 113, no. 44, pp. 14725-14731, 2009.

[8] F. N. Mendoza, R. Lopez-Rendon, J. Lopez-Lemus, J. Cruz, and J. Alejandre, "Surface tension of hydrocarbon chains at the liquid-vapour interface," Molecular Physics, vol. 106, no. 8, pp. 1055-1059, 2008.

[9] J. Benet, L. G. MacDowell, and C. Menduiña, "Liquid-vapor phase equilibria and surface tension of ethane as predicted by the TraPPE and OPLS models," Journal of Chemical and Engineering Data, vol. 55, no. 12, pp. 5465-5470, 2010.

[10] A. E. Ismail, M. Tsige, P. J. In'T Veld, and G. S. Grest, "Surface tension of normal and branched alkanes," Molecular Physics, vol. 105, no. 23-24, pp. 3155-3163, 2007.

[11] J. T. Fern, D. J. Keffer, and W. V. Steele, "Measuring coexisting densities from a two-phase molecular dynamics simulation by voronoi tessellations," The Journal of Physical Chemistry B, vol. 111, no. 13, pp. 3469-3475, 2007.

[12] J. L. Rivera, J. Alejandre, S. K. Nath, and J. J. De Pablo, "Thermodynamic and transport properties of nitrogen and 
butane mixtures," Molecular Physics, vol. 98, no. 1, pp. 43-55, 2000.

[13] F. M. Juárez-Guerra, J. L. Rivera, A. Zúñiga-Moreno, L. A. Galicia-Luna, J. L. Rico, and J. Lara, "Molecular modeling of thiophene in the vapor-liquid equilibrium," Separation Science and Technology, vol. 41, no. 2, pp. 261-281, 2006.

[14] J. L. Rivera, F. W. Starr, P. Paricaud, and P. T. Cummings, "Polarizable contributions to the surface tension of liquid water," Journal of Chemical Physics, vol. 125, no. 9, 2006.

[15] W. L. Jorgensen, D. S. Maxwell, and J. Tirado-Rives, "Development and testing of the OPLS all-atom force field on conformational energetics and properties of organic liquids," Journal of the American Chemical Society, vol. 118, no. 45, pp. 11225-11236, 1996.

[16] C. D. Wick, M. G. Martin, and J. I. Siepmann, “Transferable Potentials for Phase Equilibria. 4. United-Atom description of linear and branched alkenes and alkylbenzenes," The Journal of Physical Chemistry B, vol. 104, no. 33, pp. 8008-8016, 2000.

[17] Z. H. Rao, S. F. Wang, and F. F. Peng, "Self diffusion and heat capacity of n-alkanes based phase change materials: a molecular dynamics study," International Journal of Heat and Mass Transfer, vol. 64, pp. 581-589, 2013.

[18] H. K. Chilukoti, G. Kikugawa, and T. Ohara, "A molecular dynamics study on transport properties and structure at the liquid-vapor interfaces of alkanes," International Journal of Heat and Mass Transfer, vol. 59, no. 1, pp. 144-154, 2013.

[19] J. L. Rivera and J. Alejandre, "Transport properties of nitrogen and $n$-alkane binary mixtures," Fluid Phase Equilibria, vol. 185, no. 1-2, pp. 389-396, 2001.

[20] S. J. Nose, "A unified formulation of the constant temperature molecular dynamics methods," The Journal of Chemical Physics, vol. 81, no. 1, pp. 511-519, 1984.

[21] A. Trokhymchuk and J. Alejandre, "Computer simulations of liquid/vapor interface in Lennard-Jones fluids: some questions and answers," Journal of Chemical Physics, vol. 111, no. 18, pp. 8510-8523, 1999.

[22] J. Dunkel and S. Hilbert, "Phase transitions in small systems: microcanonical vs. canonical ensembles," Physica A, vol. 370, no. 2, pp. 390-406, 2006.

[23] J. L. Rivera, M. Predota, A. A. Chialvo, and P. T. Cummings, "Vapor-liquid equilibrium stimulations of the SCPDP model of water," Chemical Physics Letters, vol. 357, no. 3-4, pp. 189-194, 2002.

[24] M. Ison, P. Balenzuela, A. Bonasera, and C. O. Dorso, "Dynamical properties of constrained drops," The European Physical Journal A, vol. 14, no. 4, pp. 451-457, 2002. 

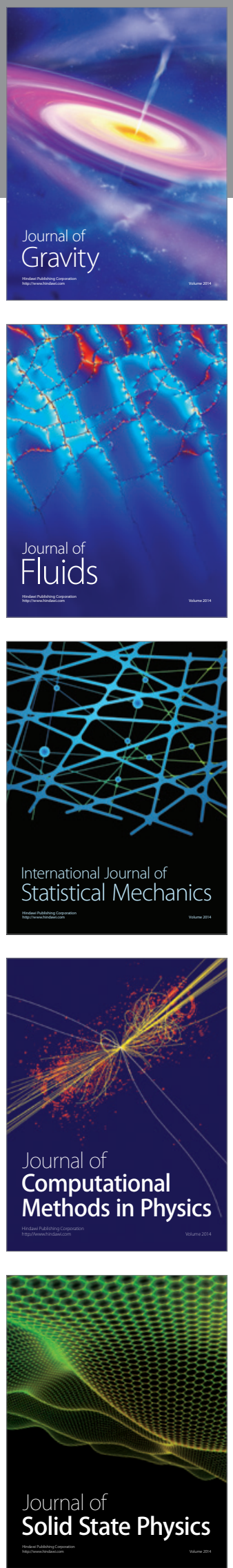

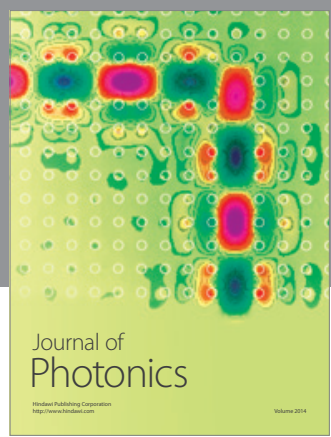

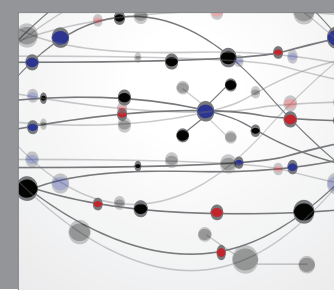

The Scientific World Journal

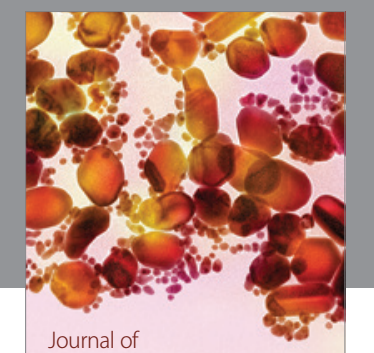

Soft Matter
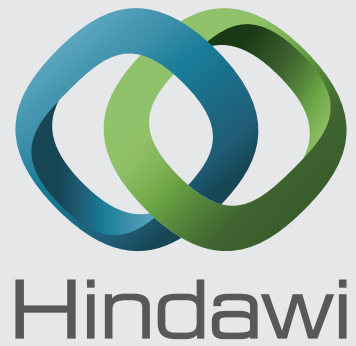

Submit your manuscripts at

http://www.hindawi.com
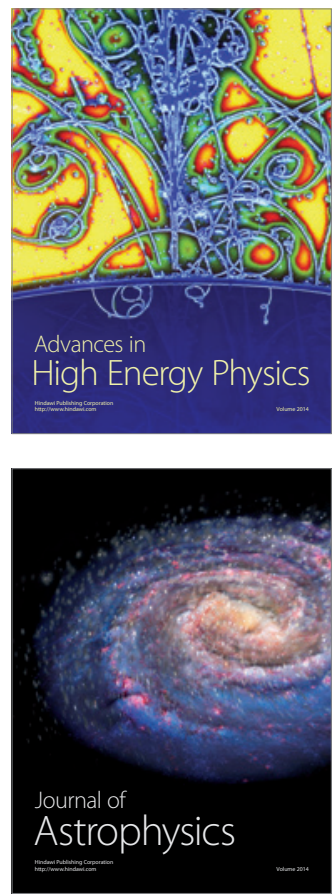
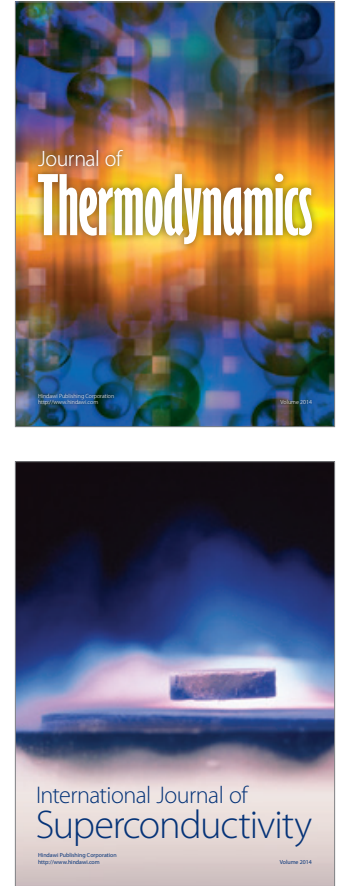
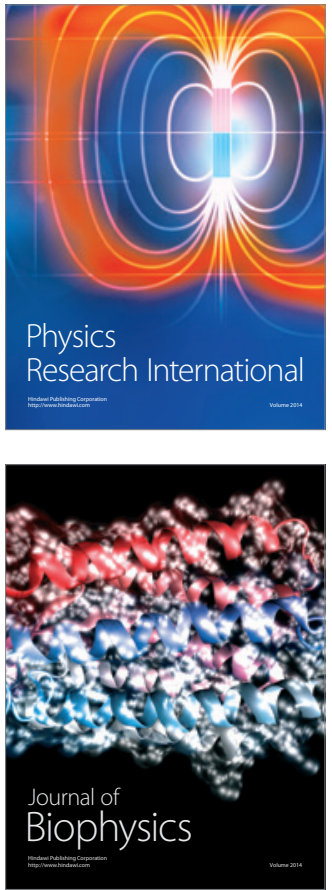
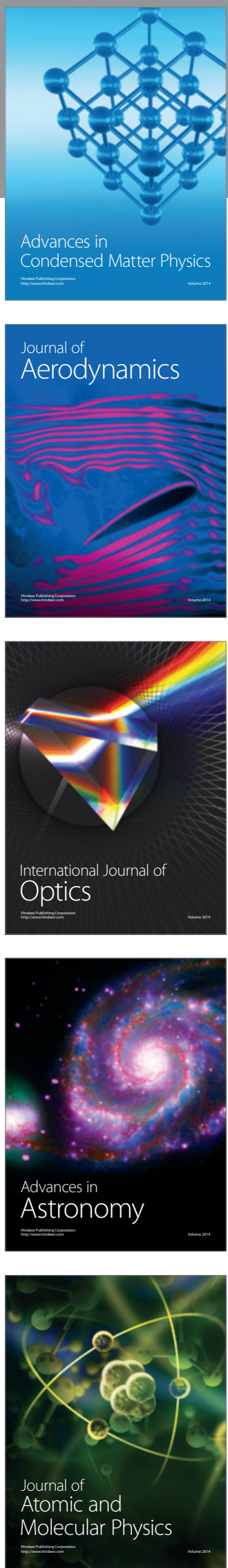